Abstract 466 Table 2 Logistic regression analysis of variables versus LNM

\begin{tabular}{|c|c|c|c|c|c|c|}
\hline & \multicolumn{3}{|c|}{ Univariate analysis } & \multicolumn{3}{|c|}{ Multivariate analysis } \\
\hline & $P$ value & Adjusted OR & $95 \% \mathrm{Cl}$ & $P$ value & Adjusted OR & $95 \% \mathrm{Cl}$ \\
\hline Age $>65$ years * & 0.946 & 0.98 & $0.47-2.01$ & - & - & - \\
\hline BMI $>30 \mathrm{~kg} / \mathrm{m}^{2}$ * & 0.710 & 1.15 & $0.54-2.45$ & - & - & - \\
\hline CA125 >35 U/mL & $<0.001$ & 8.322 & $3.71-18.67$ & $<0.001$ & 6.00 & $2.22-16.25$ \\
\hline Imaging suspected LNM & 0.001 & 4.17 & $1.77-9.86$ & 0.405 & 1.64 & $0.51-5.27$ \\
\hline $\mathrm{MI}>\mathbf{5 0} \%$ & $<0.001$ & 5.27 & $2.23-12.46$ & 0.012 & 3.64 & $1.33-10.01$ \\
\hline Cervical involvement & $<0.001$ & 14.25 & $5.37-38.04$ & $<0.001$ & 9.57 & $3.09-29.63$ \\
\hline
\end{tabular}

OR: odds ratio. Cl: confidence interval for adjusted OR. * = not included for multivariate analysis.

with normal CA125 $(\mathrm{p}<0.05)$. For patients with elevated CA125 who underwent surgical staging $(n=192)$, the prevalence of LNM was $56.5 \%$ (39/69), compared to $14.6 \%(18 /$ $123)$ in patients with normal CA125 $(\mathrm{p}<0.05)$. For patients with preoperative CT imaging $(n=148)$, LNM were suspected in $18.9 \%(28 / 148)$, but histologically confirmed in $27.7 \%$ (41/148) of the patients. Preoperative CA125 and CT findings for LNM in relation to risk of LNM are shown in table 1.

Multivariate analysis (table 2) showed that elevated CA125, histological deep $(>50 \%)$ myometrial invasion, and cervical involvement independently predict histological LNM $(\mathrm{p}<0.05$ for all) whereas positive CT findings for LNM did not.

Conclusion* This study demonstrates that elevated CA125 in patients with high-grade EC is an important prognostic marker for the predication of LNM and advanced stage disease. In patients with preoperative normal CA125, the additional value of CT imaging was limited with respect to the prediction of LNM. We therefore recommend to incorporate CA125 in routine preoperative work-up for risk stratification in high-grade EC.

\section{A PROSPECTIVE MULTICENTER NON-INFERIORITY TRIAL: ONCOLOGICAL SAFETY OF BIOPSY OF SENTINEL LYMPH NODE IN STAGE I ENDOMETRIOID ENDOMETRIAL CANCER}

${ }^{1} \mathrm{O}$ Bubliieva*, ${ }^{1} \mathrm{~V}$ Svintsitskiy, ${ }^{2} \mathrm{~S}$ Kopetskyi, ${ }^{1} \mathrm{~N}$ Tsip, ${ }^{1} \mathrm{~S}$ Nespryadko. ${ }^{1}$ National cancer institute, Oncogynaecology, Ukraine; ${ }^{2}$ National cancer institute, Department of Hepatopancreatobiliary and Oncovascular Surgery, Kyiv, Ukraine

\subsection{6/ijgc-2021-ESG0.153}

Introduction/Background* In the primary treatment of stage I intermediate risk and high-intermediate risk endometrial cancer (EC) sentinel lymph node biopsy (SLN) can be considered for staging. Although there is no prospective trials for assessment the oncological outcome in these groups.

Study hypothesis we hypothesize that progression-free survival in women who underwent biopsy of mapping sentinel lymph node with indocyanine green will be not inferior compared to the patients in which systematic lymph nodes dissection will be performed.

Methodology Trial design:This prospective, randomized, noninferiority study is to evaluate progression-free survival in patients with endometrioid EC with stage I from the intermediate and high-intermediate risk groups, who will have been performed SLN biopsy and systemic lymphadenectomy (pelvic and para-aortic lymph nodes dissection).
Inclusion criteria: 1.histological confirm endometrioid cancer of endometrium. 2. intermediate and high-intermediate risk (grade 1 , grade 3 with $\geq 50 \%$ myometrial invasion, grade 3 with $<50 \%$ myometrial invasion).

Exclusion criteria: 1 . low risk (G1 with with $<50 \%$ myometrial invasion), 2. other histological tumor variant except for endometrioid.

Result(s)* Primary endpoint: 1. Progression-free survival in patients with isolated tumor cells 2. Quality of life by EORTC QLQ-C30 and LYMQOL-Leg scales 3. 30-day post-surgery complications by Clavien-Dindo scale 4. overall survival rate which is defined from the moment of randomization to death (any cause); alive patients are analyzed after acquisition of the last information

Secondary endpoints: progression-free survival in patients with endometrioid stage I intermediate and high-intermediate risk endometrial cancer

Sample size: 430 patients for study cohort

Conclusion* Estimated Dates for Completing Accrual and Presenting Results : the study will be completed in 2023 with results in 2026

Trial Registration Number of local ethical protocol in Ukraine - №163 (23.06.20)

Disclosures Authors declare no disclosures.

\section{ENDOMETRIAL CANCER - THE SURGICAL TREATMENT IN THE HANDS OF RESIDENTS OF ONCOLOGICAL GYNAECOLOGY SUPERVISED BY SPECIALISTS}

M Mrugała*, K Nowak, A Machnicka-Rusek, M Kalus, J Tomiczek-Siwiec, B Samborska, Z Borowiec, E Milnerowicz-Nabzdyk. SPZOZ Opolskie Centrum Onkologii im. prof. Tadeusza Koszarowskiego, Opole, Poland

\subsection{6/ijgc-2021-ESG0.154}

Introduction/Background* Endometrial cancer is the most common of gynaecological malignant neoplasms in Europe and developed countries. Early diagnosis guarantees higher chances of recovery and the characteristics of this cancer allow us to treat it in a minimally invasive way which brings many benefits for the women.

This is the reason for such a big significance of excellent training in laparoscopic and robotic surgery amongst trainees in Gynaecological Oncology.

Methodology The patients with diagnosed endometrial cancer in FIGO stages I and II were sequentially assigned to two groups to gather 50 of each: laparoscopic and open surgery. The open procedures were performed by teams comprised of 2 doctors and the laparoscopic were performed by 3 doctors 
in the team. All of the surgeries were carried out by trainees in Gynaecological Oncology and supervised by a specialist. The qualifications for a total hysterectomy with bilateral salpingo-oophorectomy and lymph node staging were based on ESGO recommendations. Two groups were compared in the following fields: age, BMI, time of the procedure, total number of dissected pelvic and paraaortic lymph nodes, complications, postoperative hospital stay.

Result(s)* Most of the results obtained were similar in both groups. The average age of the participants in both groups was 64,57 years (laparoscopy: 63,48 years, open surgery: 66,35 years), BMI was $31,49 \mathrm{~kg} / \mathrm{m} 2$ (31,67 vs $31,25 \mathrm{~kg} / \mathrm{m} 2$ ). The quantity of dissected lymph nodes during laparoscopy was 21,2 pelvic nodes and 12,4 paraaortic nodes per procedure and respectively 23,76 and 12,9 for laparotomy. The rate of complications was equal in both groups $(n=6)$. Significant differences were observed in two areas. The time of surgery was longer in a laparoscopic group (181 minutes) vs. 141 minutes for open surgery. The postoperative stay took 3 days after the minimally invasive procedure and 6 days after laparotomy.

Conclusion* The outcome of surgical treatment performed by trainees was comparable in both groups. The minimally invasive surgery group had many benefits as quick recovery, great precision of surgery with an adequate oncological accuracy. The longer duration of laparoscopic procedures is a result of a learning curve.

\section{SURVIVAL ANALYSIS OF ENDOMETRIAL CANCER. OUR EXPERIENCE IN LAS PALMAS DE GRAN CANARIA}

AF Rave Ramirez, D González García-Cano, M Laseca Modrego, O Arencibia Sanchez, L Molero Sala, A Martín Martínez. Complejo Hospitalario Universitario Insular Materno Infantil de Gran Canaria, Gynecologic Oncology, las palmas de gran canaria, Spain

\subsection{6/ijgc-2021-ESGO.155}

Introduction/Background* Self-evaluation and analysis of healthcare practice in a Gynecology Oncology unit represents an advance in the strategy to improve the quality of cancer care, in this case,endometrial cancer, allowing to obtain relevant data on the quality of care practice.

Methodology Retrospective descriptive study carried out in CHUIMI. We included patients diagnosed with endometrial cancer during the study period 2012-2016. We analyzed epidemiological variables, histological type, type of treatment, final FIGO stage and 5 -year overall survival.

Result(s)* A total of 498 patients were diagnosed with endometrial cancer during this period. Mean age of 64.3 years (range 29-89 years) and a mean BMI of 33.5. 60.2\% of the patients were diagnosed in stage IA; $48.7 \%$ of which were grade 1 and 2. Type I (endometrioid and mucinous) accounted for $74.3 \%$ versus $25.7 \%$ type II. $40.8 \%$ of the patients were classified as low risk presenting a 5 -year survival of $99 \%$. Patients with intermediate risk represented $5.9 \%$ and had a 96\% 5-year survival. Patients with intermediate-high risk $(19.7 \%)$ had a 5 -year survival of $88 \%$. The patients included in the high-risk group $(28.2 \%)$, had a 5 -year survival of $62 \%$, these differences were statistically significant.

We analyzed survival according to the histological type, finding a 5-year overall survival rate of $91.9 \%$ in type I, while it was $60.8 \%$ fot type II, these differences were statistically significant.
Conclusion* The data analyzed in our Gynecology Oncology Unit are similar to those exposed in the literature, there is an important difference in terms of 5 -year survival between the low and high risk groups, $99 \%$ vs $62 \%$ respectively.

\section{TERTIARY LYMPHOID STRUCTURES AS MARKERS OF ANTI-TUMOR IMMUNITY WITH INDEPENDENT PROGNOSTIC VALUE IN THE PORTEC-3 TRIAL OF HIGH- RISK ENDOMETRIAL CANCER}

${ }^{1} \mathrm{~N}$ Horeweg, ${ }^{2} \mathrm{H}$ Workel, ${ }^{3} \mathrm{D}$ Loiero, ${ }^{4} \mathrm{D}$ Church, ${ }^{5} \mathrm{~L}$ Vermij, ${ }^{5} \mathrm{~A}$ Leon-Castillo, ${ }^{1} \mathrm{~S}$ De Boer, ${ }^{1 ; 6} \mathrm{R}$ Nout, ${ }^{7} \mathrm{M}$ Powell, ${ }^{8} \mathrm{~L}$ Mileshkin, ${ }^{9} \mathrm{H}$ Mackay, ${ }^{10} \mathrm{~A}$ Leary, ${ }^{11} \mathrm{~N}$ Singh, ${ }^{12}$ I JürgenliemkSchulz, ${ }^{1} \mathrm{CL}$ Creutzberg, ${ }^{3 ; 13} \mathrm{~V}$ Kölzer, ${ }^{2} \mathrm{HW}$ Nijman, ${ }^{14} \mathrm{~T}$ Bosse, ${ }^{2} \mathrm{M}$ De Bruyn*. ${ }^{1}$ Leiden University Medical Center (LUMC), Department of Radiation Oncology, Leiden, Netherlands; 2University Medical Center Groningen, Department of Obstetrics and Gynaecology Gynaecological Oncology , Groningen, Netherlands; ${ }^{3}$ University Hospital of Zürich, Department of Pathology and Molecular Pathology, Zürich, Switzerland; ${ }^{4}$ Wellcome Trust Centre for Human Genetics, UK; ${ }^{5}$ Leiden University Medical Center (LUMC), Department of Pathology, Leiden, Netherlands; ${ }^{6}$ Erasmus MC Cancer Institute, Department of Radiotherapy, Rotterdam, Netherlands; 'Barts Health NHS Trust, Department of Clinical Oncology, UK; ${ }^{8}$ Peter MacCallum Cancer Centre, Department of Medical Oncology, Melbourne, Australia; ${ }^{9}$ Sunnybrook Health Sciences Centre -Odette Cancer Centre, Division of Medical Oncology and Haematology, Toronto, Canada; ${ }^{10}$ Institute Gustave Roussy, Department of Medical Oncology, Villejuif, France; ${ }^{11}$ Barts Health NHS Trust, Department of Pathology, UK; ${ }^{12}$ UMC Utrecht, Department of Radiation Oncology, Utrecht, Netherlands; ${ }^{13}$ University of Oxford, Department of Oncology and Nuffield Department of Medicine, UK; ${ }^{14}$ Leiden University Medical Center, Department of Pathology, Leiden, Netherlands

\subsection{6/ijgc-2021-ESG0.156}

Introduction/Background* Tertiary lymphoid structures (TLS) are ectopic lymphoid tissues that form in and around cancers. TLS consist of a germinal centre (GC) with proliferating B-cells and follicular dendritic cells (FDCs), as well as a peripheral Tcell zone. Local and systemic B- and T-cell responses are thought to be initiated and maintained at the TLS. Here, we analysed whether TLS were associated with anti-tumour immunity and a reduced risk of recurrence in endometrial cancer (EC). Methodology TLS were quantified by an expert pathologist (TB) on H\&E-stained tumour slides of the cancer genome atlas uterine cancer cohort (TCGA UCEC), and by immunohistochemistry (IHC) on tumour slides from the PORTEC-3 trial biobank. Time to recurrence analysis were performed according to Kaplan-Meier's method, using log-rank tests and Cox' proportional hazards models, including prespecified multivariable analysis with clinicopathological and molecular risk factors.

Result(s)* Differential gene expression analysis of TLS-positive and TLS-negative cases from TCGA UCEC identified, among others, L1-cell adhesion molecule (L1CAM) (figure 1A). IHC of PORTEC-3 cases revealed expression of L1CAM in TLS GCs, where it co-localised with CD21 on FDCs. TLS were observed in the myometrial wall distal and proximal to the tumour (figure 1B). Tumour tissues of 377 PORTEC-3 participants could be included for analysis of L1CAM-defined TLS. L1CAM-defined TLS were identified independent of L1CAM expression in tumour cells and across all molecular subgroups, though enriched in mismatch repair deficient (MMRd) and polymerase-epsilon mutant (POLEmut) EC (figure 2). TLS were significantly more frequent in MMRd EC with secondary TP53 mutations $(p=0.008)$. Intra-tumoural $\mathrm{CD}^{+}{ }^{+}$-cell densities were significantly higher in TLS-positive cases. Five-year risk of recurrence was $7.2 \%(95 \% \mathrm{CI} 0.9-13.1)$ in EC patients with TLS ( $n=70)$, and 27.8\% (95\%CI 22.6-32.7, p-value <0.0001) in EC patients without TLS $(n=307)$. This favourable 